\title{
The selenium content of SEPP1 versus selenium requirements in vertebrates
}

Sam Penglase, Kristin Hamre, Ståle Ellingsen

Selenoprotein P (SEPP1) distributes selenium (Se) throughout the body via the circulatory system. For vertebrates, the Se content of SEPP1 varies from 7 to 18 Se atoms depending on the species, but the reason for this variation remains unclear. Herein we provide evidence that vertebrate SEPP1 Sec content correlates positively with Se requirements. As the Se content of full length SEPP1 is genetically determined, this presents a unique case where a nutrient requirement can be predicted based on genomic sequence information. 
1 The selenium content of SEPP1 versus selenium requirements in vertebrates.

2 S. Penglase ${ }^{1,2,3}$, K. Hamre ${ }^{1}$ and S. Ellingsen ${ }^{1}$

$3 \quad{ }^{1}$ National Institute of Nutrition and Seafood Research (NIFES), PO Box 2029, NO-5817 Bergen,

4 Norway

$5 \quad 2$ Department of Biology, University of Bergen, PO Box 7803, NO-5020 Bergen, Norway

$6{ }^{3}$ Current address: Aquaculture Research Solutions (ARS), 44 Mango Avenue, Mundingburra, 7 4812, Australia

8

9

10

11

12 Corresponding Author

13 PhD Sam Penglase

14 ARS, 44 Mango Avenue, Mundingburra, 4812, QLD, Australia

15 Telephone: +61439529434

16 E-mail: sampenglase@hotmail.com 


\section{Abstract}

18 Selenoprotein P (SEPP1) distributes selenium (Se) throughout the body via the circulatory

19 system. For vertebrates, the Se content of SEPP1 varies from 7 to 18 Se atoms depending on the

20 species, but the reason for this variation remains unclear. Herein we provide evidence that

21 vertebrate SEPP1 Sec content correlates positively with Se requirements. As the Se content of

22 full length SEPP1 is genetically determined, this presents a unique case where a nutrient

23 requirement can be predicted based on genomic sequence information. 


\section{Introduction}

Selenium (Se) is an essential trace element required for selenocysteine ( $\mathrm{Sec}$ ) residues inserted during mRNA translation into Se dependent proteins, termed selenoproteins (Brigelius-Flohé 1999). Selenocysteine is a relatively rare Se containing analogue of the essential amino acid cysteine (Cys) (Papp et al. 2007, Penglase 2014). The number of genes coding for selenoproteins varies among species, with mammals having 24 to 25, birds 25, and bony fish 35 to 38 (Mariotti et al. 2012). Most selenoproteins are redox enzymes that contain a single Se atom present within a catalytically active Sec residue (Papp et al. 2007). An exception is the Se rich glycoprotein, selenoprotein P (SEPP1; aka SeP, SEPP, SEPP1a), which in vertebrates contains 7 to 18 Sec residues, depending on the species (Lobanov et al. 2008). The high Sec content of SEPP1 is thought to facilitate Se distribution throughout the body. In mammals, the liver is a major site of SEPP1 expression, where it is synthesised utilising Se obtained from food. Hepatic SEPP1 is then secreted into the blood plasma (Kato et al. 1992). Of the Se that is present in the bioavailable pool, plasma SEPP1 accounts for around $80 \%$ of the total Se in plasma (Hill et al. 1996, Hill et al. 2007), and $8 \%$ of the total body Se (Read et al. 1990). Tissues utilise a combination of receptor mediated endocytosis and pinocytosis to obtain SEPP1 from the plasma, where it is then catabolised to release Se for de nova selenoprotein synthesis (Burk and Hill 2009, Burk et al. 2013).

Several features of SEPP1 are conserved among vertebrates including, i) a single N-terminal domain Sec residue present within a thioredoxin like motif (UXXC, where $U$ is Sec), ii) a histidine rich region in the mid region of the protein, and iii) an apolipoprotein $\underline{E} \underline{\text { receptor- } \underline{2}}$ (APOER2; aka LRP8) binding site followed by five Sec residues in proximity to the C-terminal 
47 (Fig. 1) (Lobanov et al. 2008). APOER2 is widely expressed in human tissues 48 (www.humanproteomemap.org; (Kim et al. 2014). APOER2 facilitated uptake of plasma SEPP1 49 is an essential (testes) or important (brain and foetus) pathway in some, but not all (muscle, 50 kidney, liver or whole body) tissues for maintaining Se homeostasis in vivo (Burk et al. 2007, 51 Olson et al. 2007, Hill et al. 2012, Burk et al. 2013). In contrast, the histidine rich regions of 52 SEPP1 presumably interact with multiple receptors, including megalin (LRP2). A megalin facilitated uptake pathway minimises excretion of Se by binding SEPP1 fragments in the kidney endothelial cells at sites of inflammation (Hondal et al. 2001, Saito et al. 2004).

In contrast, other domains in SEPP1 have low conservation among species. For example, singlenucleotide mutations causing Sec to cysteine (Cys) substitutions in the SEPP1 C-terminal domain upstream and including the APOER2 binding site have occurred frequently throughout the vertebrate linage (Fig. 1) (Lobanov et al. 2008). The reason why Sec content plasticity is observed only within this region of SEPP1 is unclear, but it appears to be responsible for most of the variation between the SEPP1 Sec content among vertebrates (Lobanov et al. 2008). Furthermore, why SEPP1 Sec content differs among species also remains unknown. Several lines of evidence suggest vertebrate SEPP1 Sec number may be a direct function of Se utilisation. For instance, vertebrate SEPP1 Sec content correlates positively with selenoproteome size, tissue Se levels, and Se bioavailability in the environment (Lobanov et al. 2008). 
71 If a direct relationship between SEPP1 Sec content and Se requirements exists, the SEPP1 Sec

content of a species could predict its Se requirements, or vice versa. In doing so, this would provide a new insight into how the genome affects nutrient utilisation. Additionally, such a relationship would allow considerable scope for implementing the 3R's (replace, reduce, refine).

For example, this relationship would indicate the dietary Se levels to focus on when investigating the Se requirements for novel species. Such knowledge would reduce both the number of animals required and the risk of exposure to Se levels that may compromise animal welfare in such experiments.

In the following work, we compared the Sec content of mammalian, avian and bony fish SEPP1s predicted in silico with their Se requirements determined in vivo. We found a strong positive non-linear correlation $\left(\mathrm{R}^{2}=0.78\right)$ between the two, suggesting Se requirements can be predicted from the Seppl gene sequence. The correlation was dictated by the Sec content within the Cterminal domain upstream and including the APOER2 binding site of SEPP1s. The model was limited, as it could not predict Se requirements in species whose SEPP1 Sec content was >15 residues, as found in the majority of bony fish species. The predicted Se requirements for vertebrate species based on their SEPP1 Sec content are provided. 
90 The in silico predicted species specific Sec content of SEPP1 (SEPP1a in fish) were obtained

91 from Lobanov et al. (2008), the open access selenoprotein database (selenodb.org; (Romagné et 92 al. 2014)) or by analysing genomic Seppl sequences (NCBI) for Sec content

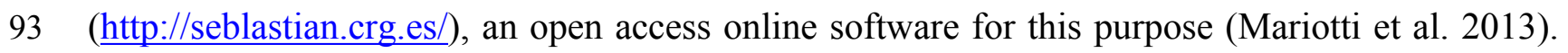

94 The SEPP1 Sec content of five bony fish species; loach (Paramisgurnus dabryanus), cobia 95 (Rachycentron canadum), grouper (Epinephelus malabaricus), gibel carp (Carassius auratus 96 gibelio) and yellowtail kingfish (Seriola lalandi); were assumed to be within the 15 to 17 residue 97 range found for fish in general (Lobanov et al. 2008)(See Supp. Table 2). Protein alignments and a phylogenetic tree for vertebrate SEPP1 are provided in Supplementary Figures 2 and 3, respectively. The species specific Se requirement data were obtained from published studies and from the National Research Council of the USA (NRC) nutrient requirement reports (NRC 1963, Hilton et al. 1980, Gatlin and Wilson 1984, NRC 1985, NRC 1994, NRC 1995, Weiss et al. 1996, NRC 1997, Weiss et al. 1997, Lei et al. 1998, Wedekind et al. 2004, Lin and Shiau 2005,

Fischer et al. 2008, Jensen and Pallauf 2008, Sunde et al. 2009, Liu et al. 2010, Sunde and Hadley 2010, Han et al. 2011, NRC 2011, Le and Fotedar 2013, Hao et al. 2014, Penglase et al. 2014). See Supp. Table 1 for further information regarding these animal Se requirement studies. Where multiple Se requirement studies for a species are available, the dietary Se requirements to 107 fulfil the requirements of the actively growing juvenile stage were selected. Data were analysed 108 in GraphPad Prism (GraphPad Software, San Diego, CA, USA, V. 5.04). Data were fitted with a 109 horizontal line (null hypothesis) and then tested against more complex models in the following

110 sequence; first order polynomial, second order polynomial and five parameter logistic equation 111 (5PL) asymmetric sigmoidal; until the simplest model that explained the data was found 
$112(\mathrm{p}<0.05)$. Other vertebrate classes (reptiles and amphibians) were excluded from the analyses 113 because of the absence of Se requirement studies. 


\section{Results and Discussion}

\section{The selenocysteine content of Selenoprotein P correlates strongly with selenium requirements.}

116 The Sec content of SEPP1s were identified for a total of 14 species; three bony fish, three birds 117 and eight mammals; for which the Se requirements are also published (Supp. Table 1). Using this 118 data, a positive non-linear correlation $\left(\mathrm{R}^{2}=0.78\right)$ was found between Se requirements and

119 SEPP1 Sec number (Fig. 2). This reflects the positive correlation between SEPP1 Sec content 120 and selenoprotein number in vertebrates found previously (Kryukov and Gladyshev 2000, 121 Lobanov et al. 2008). A linear relationship between Se requirements and SEPP1 Sec content was 122 moderately strong $\left(\mathrm{R}^{2}=0.68\right)$ but was statistically rejected $(\mathrm{p}=0.048)$ in favour of the non-linear 123 model mentioned above.

124

125 All fish annotated to date have SEPP1 (aka SEPP1a in fish) with 15 to 17 Sec residues (See 126 Supp. Table 2). Based on this, an additional five bony fish species with known Se requirements 127 were assumed to have SEPP1s with $17 \mathrm{Sec}$ residues and added to the data set, which was then re128 analysed. This resulted in an asymmetric sigmoidal trend with a plateau at 17.0 (Fig. 2), 129 suggesting that a species SEPP1 is only useful for predicting Se requirements prior to this 130 plateau ( $\leq 16$ Sec residues). When a species SEPP1 has $>16$ Sec residues, as is found in many 131 fish species, this curve predicts a minimum requirement $(0.24 \mathrm{mg} / \mathrm{Se} \mathrm{kg}$ dry matter (DM)) but 132 not a maximum (there is no correlation between SEPP1 Sec content and Se requirements above 133 this level). Modelling the data with alternative SEPP1 Sec content (15 or 16 Sec) for these five 134 fish species shifts the plateau height towards those values, but retains the general features of the model. The asymmetric sigmoidal model (Fig. 2, segmented line) differs from the second order 
136 polynomial model (Fig. 2, solid line), which only predicts Se requirements for species with

137 SEPP1s containing up to $15 \mathrm{Sec}$ residues $(0.20 \mathrm{mg} / \mathrm{Se} \mathrm{kg}$, Table 1$)$.

138 The model (Fig. 2) demonstrates the broad range of Se requirements found for bony fish ( 0.25 to

$1395.56 \mathrm{mg} \mathrm{Se} / \mathrm{kg}$ dry feed) that occurs over a small range of SEPP1 Sec contents (15 to $17 \mathrm{Sec}$

140 residues). The reason/s for this are unknown. Limitations to increasing SEPP1 Sec content above

14117 residues may have led fish to utilise regulatory mechanisms to increase Se supply to

142 peripheral tissues. For example Sepp 1 mRNA expression is elevated in fish, particularly in the

143 kidneys, in comparison to mammals (Lobanov et al. 2008). This suggests plasma SEPP1 in fish

144 may be replenished by SEPP1 synthesised from Se scavenged in the kidneys. On the other hand,

145 the single nucleotide mutation required to change a Sec to a Cys codon (Lobanov et al. 2008)

146 may have allowed mammals to decrease SEPP1 Sec content in line with Se requirements,

147 resulting in the large range of SEPP1 Sec contents (7 to $15 \mathrm{Sec}$ residues) found in mammals. The

148 Se requirements versus SEPP1 Sec content in vertebrates predicted by the second order

149 polynomial model (Fig. 2, solid line) are provided in Table 1.

150 It is essential to note that the correlation between SEPP1 Sec content and Se requirements does

151 not prove causation. Another factor/s may be involved in the simultaneous increase in SEPP1

152 Sec content and Se requirements observed in this study, such as the environmental availability of

153 Se. For example, within vertebrate classes, species with Sec poor SEPP1s are often found in

154 habitats with lower background levels of Se. Both guinea pigs and naked mole rats

155 (Heterocephalus glaber) have Sec poor SEPP1s (7 residues), low Se requirements (Jensen and

156 Pallauf 2008, Kasaikina et al. 2011) and inhabit the Andes or East Africa respectively, both

157 regions of low Se status (FAO 1992, Rachel et al. 2013). Freshwater habitats often have lower 
158 background levels of Se than marine habitats (Combs and Combs 1986, Santos et al. 2015) and

159 freshwater fish have on average less Sec in SEPP1 than marine fish (Supp. Table 2).

160 Furthermore, SEPP1 appears to have originated in invertebrates, but thus far SEPP1 (along with

161 greater number of selenoproteins), has only been found in invertebrates inhabiting marine

162 environments (Lobanov et al. 2009, Liang et al. 2012). Added to this, if a direct relationship does

163 exist between SEPP1 Sec content and Se requirements, it is unclear which factor is causing the 164 other.

166 Overall, we hypothesise that environmental Se availability was an evolutionary pressure to

167 decrease Se utilisation as animals progressed from Se rich marine environments into fresh water 168 and terrestrial habitats where environmental Se levels are generally lower. Selection then 169 occurred for decreased Se utilisation (Se requirements), which resulted in decreased selection 170 pressure on maintaining, and then decreases in, SEPP1 Sec number. The results were new 171 species-specific equilibriums between environmental Se availabilities, Se requirements and 172 SEPP1 Sec contents.

173

A hypothesis for the Sec number plasticity or conservation in different domains of vertebrate

175 SEPP1.

176 As discussed, most of the difference in the SEPP1 Sec content between species is a result of 177 differences in the Sec content found upstream and including the APOER2 binding site within the 178 C-domain of SEPP1 (Fig. 1 and Supp. Table 2). When we analysed the Sec content in this region 179 in relation to a species Se requirement (Supp. Fig. 1), we found a similar positive correlation as 180 found for full-length SEPP1 and Se requirements (Fig. 2), supporting this statement. Recently it 
181 was found that SEPP1 Sec residues closer to the C-terminal are translated with greater efficiency

182 than those towards the N-terminal (Shetty et al. 2014). Premature termination of SEPP1

183 translation at Sec codons appears to be a common event. For instance, four rat SEPP1 isoforms

184 have been identified in plasma, whereby in addition to the full length protein, shorter variants are

185 synthesised when translation is terminated at the second, third or seventh Sec codon (Ma et al.

186 2002). Thus, on average each plasma SEPP1 in mice contains 5 Sec residues, not the 10 Sec

187 residues expected if only the full length protein is present (Hill et al. 2007). As a consequence of

188 this, a proportion of translated SEPP1 proteins will not contain the APOER2 binding site (Fig.

189 1).

190

191 Thus, as discussed we hypothesise that decreases in Se requirements are an evolutionary

192 adaption to Se availability. Secondly, we hypothesise that the Se requirements of the brain

193 among species is similar on a weight basis, despite differences in the Se requirements of the

194 whole body. For instance, compared to mice, naked mole rats have lower levels (-30 to $-75 \%)$ of

195 Se in most tissues except the brain (Kasaikina et al. 2011). And lastly, low Se availability can

196 stall translation of selenoproteins at Sec codons (Weiss Sachdev and Sunde 2001), and may be a

197 reason for the truncated forms of SEPP1 translated in vivo. Thus Sec to Cys substitutions in

198 SEPP1 may have occurred specifically in the region downstream and including the APOER2

199 binding site as it aids the translation of full-length protein under Se limiting conditions, such as

200 those faced by naked mole rats and guinea pigs. The subsequent retention of the APOER2

201 binding site would allow the continuation of a controlled Se supply to critical organs, such as the

202 brain, that utilise APOER2 mediated uptake of SEPP1. 


\section{Conclusion}

205

206

207

208

209

210

211

212

213

214

215

216

217

218

219

220

221

222

223

224

225

226

227

228

229

230

231

232

233

234

235

236

The Sec content of SEPP1 correlates with Se requirements in vertebrates with $\leq 15$ Sec residue SEPP1s. No correlation occurred between SEPP1 Sec content and Se requirements for species with $>15 \mathrm{Sec}$ residue SEPP1s; however, a minimum Se requirement of $0.20 \mathrm{mg} \mathrm{Se} / \mathrm{kg}$ DM for these species was predicted. This study suggests that genome evolution is affected directly by nutrient availability in the environment, and provides novel evidence that the genomic sequence can be used to predict a nutrient requirement.

\section{Acknowledgements}

We would like to extend our thanks to Dr Sofia Fortunato for her technical expertise on protein alignments and phylogenetic tree analyses.

\section{References}

Brigelius-Flohé, R. (1999). "Tissue-specific functions of individual glutathione peroxidases." Free Radical Biology and Medicine 27(9-10): 951-965.

Burk, R. F. and K. E. Hill (2009). "Selenoprotein P - Expression, functions, and roles in mammals." Biochimica et Biophysica Acta (BBA) - General Subjects 1790(11): 1441-1447.

Burk, R. F., K. E. Hill, G. E. Olson, E. J. Weeber, A. K. Motley, V. P. Winfrey and L. M. Austin (2007). "Deletion of apolipoprotein E receptor-2 in mice lowers brain selenium and causes severe neurological dysfunction and death when a low-selenium diet is fed." Journal of Neuroscience 27(23): 6207-6211.

Burk, R. F., G. E. Olson, K. E. Hill, V. P. Winfrey, A. K. Motley and S. Kurokawa (2013). "Maternal-fetal transfer of selenium in the mouse." FASEB journal 27(8): 3249-3256.

Chiu-Ugalde, J., F. Theilig, T. Behrends, J. Drebes, C. Sieland, P. Subbarayal, J. Köhrle, A. Hammes, L. Schomburg and U. Schweizer (2010). "Mutation of megalin leads to urinary loss of selenoprotein P and selenium deficiency in serum, liver, kidneys and brain." The Biochemical journal 431(1): 103-111.

Combs, G. F. and S. B. Combs (1986). Selenium in foods and feeds. The role of selenium in nutrition. G. F. Combs and S. B. Combs. New York, Academic Press Inc: 41-126.

FAO, Ed. (1992). Status of Cadmium, Lead, Cobalt and Selenium in Soils and Plants of Thirty Countries. FAO Soils Bulletin. Forrsa, Finland.

Fischer, J., A. Bosse, E. Most, A. Mueller and J. Pallauf (2008). "Selenium requirement of growing male turkeys." British poultry science 49(5): 583-591.

Gatlin, D. M., III and R. P. Wilson (1984). "Dietary selenium requirement of fingerling channel catfish." Journal of Nutrition 114(3): 627-633. 
Han, D., S. Xie, M. Liu, X. Xiao, H. Liu, X. Zhu and Y. Yang (2011). "The effects of dietary selenium on

238 growth performances, oxidative stress and tissue selenium concentration of gibel carp (Carassius auratus gibelio)." Aquaculture Nutrition 17(3): e741-e749. Hao, X., Q. Ling and F. Hong (2014). "Effects of dietary selenium on the pathological changes and oxidative stress in loach (Paramisgurnus dabryanus)." Fish physiology and biochemistry. Hill, K., S. Wu, A. Motley, T. Stevenson, V. Winfrey, M. Capecchi, J. Atkins and R. Burk (2012).

"Production of selenoprotein P (Sepp1) by hepatocytes is central to selenium homeostasis." The Journal of Biological Chemistry 287(48): 40414-40424.

Hill, K. E., Y. Xia, B. Akesson, M. E. Boeglin and R. F. Burk (1996). "Selenoprotein P concentration in plasma is an index of selenium status in selenium-deficient and selenium-supplemented Chinese subjects." The Journal of nutrition 126(1): 138-145. Hill, K. E., J. Zhou, L. M. Austin, A. K. Motley, A. J. L. Ham, G. E. Olson, J. F. Atkins, R. F. Gesteland and R. F. Burk (2007). "The selenium-rich C-terminal domain of mouse selenoprotein $P$ is necessary for the supply of selenium to brain and testis but not for the maintenance of whole body selenium." Journal of Biological Chemistry.

Hilton, J. W., P. V. Hodson and S. J. Slinger (1980). "The requirement and toxicity of selenium in rainbow trout (Salmo gairdneri)." Journal of Nutrition 110: 2527-2535.

Hondal, R. J., S. Ma, R. M. Caprioli, K. E. Hill and R. F. Burk (2001). "Heparin-binding histidine and lysine residues of rat selenoprotein $P$." The Journal of biological chemistry 276(19): 15823-15831.

Jensen, C. and J. Pallauf (2008). "Estimation of the selenium requirement of growing guinea pigs (Cavia porcellus)." Journal of Animal Physiology and Animal Nutrition 92(4): 481-491.

Kasaikina, M. V., A. V. Lobanov, M. Y. Malinouski, B. C. Lee, J. Seravalli, D. E. Fomenko, A. A. Turanov, L. Finney, S. Vogt, T. J. Park, R. A. Miller, D. L. Hatfield and V. N. Gladyshev (2011). "Reduced utilization of selenium by naked mole rats due to a specific defect in Gpx1 expression." Journal of Biological Chemistry 286(19): 17005-17014.

Kato, T., R. Read, J. Rozga and R. F. Burk (1992). "Evidence for intestinal release of absorbed selenium in a form with high hepatic extraction." The American journal of physiology 262(5 Pt 1): G854-G858.

Kim, M.-S. S., S. M. Pinto, D. Getnet, R. S. Nirujogi, S. S. Manda, R. Chaerkady, A. K. Madugundu, D. S. Kelkar, R. Isserlin, S. Jain, J. K. Thomas, B. Muthusamy, P. Leal-Rojas, P. Kumar, N. A. Sahasrabuddhe, L. Balakrishnan, J. Advani, B. George, S. Renuse, L. D. Selvan, A. H. Patil, V. Nanjappa, A. Radhakrishnan, S. Prasad, T. Subbannayya, R. Raju, M. Kumar, S. K. Sreenivasamurthy, A. Marimuthu, G. J. Sathe, S. Chavan, K. K. Datta, Y. Subbannayya, A. Sahu, S. D. Yelamanchi, S. Jayaram, P. Rajagopalan, J. Sharma, K. R. Murthy, N. Syed, R. Goel, A. A. Khan, S. Ahmad, G. Dey, K. Mudgal, A. Chatterjee, T.-C. C. Huang, J. Zhong, X. Wu, P. G. Shaw, D. Freed, M. S. Zahari, K. K. Mukherjee, S. Shankar, A. Mahadevan, H. Lam, C. J. Mitchell, S. K. Shankar, P. Satishchandra, J. T. Schroeder, R. Sirdeshmukh, A. Maitra, S. D. Leach, C. G. Drake, M. K. Halushka, T. S. Prasad, R. H. Hruban, C. L. Kerr, G. D. Bader, C. A. lacobuzio-Donahue, H. Gowda and A. Pandey (2014). "A draft map of the human proteome." Nature 509(7502): 575-581.

Kryukov, G. V. and V. N. Gladyshev (2000). "Selenium metabolism in zebrafish: multiplicity of selenoprotein genes and expression of a protein containing 17 selenocysteine residues." Genes to Cells 5(12): 1049-1060.

Kurokawa, S., S. Eriksson, K. L. Rose, S. Wu, A. K. Motley, S. Hill, V. P. Winfrey, W. H. McDonald, M. R. Capecchi, J. F. Atkins, E. S. Arnér, K. E. Hill and R. F. Burk (2014). "Sepp1(UF) forms are N-terminal selenoprotein P truncations that have peroxidase activity when coupled with thioredoxin reductase-1." Free radical biology \& medicine 69: 67-76.

Le, K. T. and R. Fotedar (2013). "Dietary selenium requirement of yellowtail kingfish (Seriola lalandi)." Agricultural Sciences 4(6a): 68-75. 
Lei, X. G., H. M. Dann, D. A. Ross, W. H. Cheng, G. F. Combs and K. R. Roneker (1998). "Dietary selenium supplementation is required to support full expression of three selenium-dependent glutathione peroxidases in various tissues of weanling pigs." The Journal of nutrition 128(1): 130-135. Liang, J., N. Jiazuan and L. Qiong (2012). "Evolution of selenoproteins in the metazoan." BMC Genomics 13(1): 446.

Lin, Y. H. and S. Y. Shiau (2005). "Dietary selenium requirements of juvenile grouper, Epinephelus malabaricus." Aquaculture 250(1-2): 356-363.

Liu, K., X. J. Wang, Q. H. Ai, K. S. Mai and W. B. Zhang (2010). "Dietary selenium requirement for juvenile cobia, Rachycentron canadum L." Aquaculture Research 41(10): e594-e601.

Lobanov, A., D. Hatfield and V. Gladyshev (2008). "Reduced reliance on the trace element selenium during evolution of mammals." Genome Biology 9(3): R62.

Lobanov, A. V., D. L. Hatfield and V. N. Gladyshev (2009). "Eukaryotic selenoproteins and selenoproteomes." Biochimica et biophysica acta 1790(11): 1424-1428. rat selenoprotein $\mathrm{P}$ and three isoforms shortened at the $\mathrm{C}$ terminus. Evidence that three UGA codons in the mRNA open reading frame have alternative functions of specifying selenocysteine insertion or translation termination." The Journal of biological chemistry 277(15): 12749-12754.

Mariotti, M., A. V. Lobanov, R. Guigo and V. N. Gladyshev (2013). "SECISearch3 and Seblastian: new tools for prediction of SECIS elements and selenoproteins." Nucleic acids research 41(15): e149.

Mariotti, M., P. G. Ridge, Y. Zhang, A. V. Lobanov, T. H. Pringle, R. Guigo, D. L. Hatfield and V. N. Gladyshev (2012). "Composition and evolution of the vertebrate and mammalian selenoproteomes." PLOS ONE 7(3): e33066.

NRC (1963). Nutrient requirements of beef cattle. Washington DC, National Academy Press. NRC (1985). Nutrient requirements of sheep. Washington DC, National Academy Press.

NRC (1994). Nutrient requirements of Poultry. NRC, National Academy Press, Washington, DC. NRC (1995). Nutrient requirements of laboratory animals. Washington DC, National Academy Press. NRC (1997). Nutrient requirements of horses. Washington DC, National Academy Press. NRC (2011). Nutrient requirements of fish and shrimp. Washington DC, National Academy Press. Olson, G. E., V. P. Winfrey, K. E. Hill and R. F. Burk (2008). "Megalin mediates selenoprotein P uptake by kidney proximal tubule epithelial cells." The Journal of biological chemistry 283(11): 6854-6860. Olson, G. E., V. P. Winfrey, S. K. NagDas, K. E. Hill and R. F. Burk (2007). "Apolipoprotein E receptor-2 (ApoER2) mediates selenium uptake from selenoprotein $P$ by the mouse testis." Journal of Biological Chemistry 282(16): 12290-12297.

Papp, L. V., J. Lu, A. Holmgren and K. K. Khanna (2007). "From selenium to selenoproteins: synthesis, identity, and their role in human health." Antioxidants \& Redox Signaling 9(7): 775-806.

318 Penglase, S. (2014). The effects of selenium on methylmercury toxicity in zebrafish. PhD, University of 319 Bergen.

320 Penglase, S., K. Hamre, J. D. Rasinger and S. Ellingsen (2014). "Selenium status affects selenoprotein expression, reproduction, and F1 generation locomotor activity in zebrafish (Danio rerio)." British Journal of Nutrition 111(11): 1918-1931.

Rachel, H., W. P. S. Edwin, D. Y. Scott, D. C. C. Allan, J. M. J. Edward, R. B. Colin, E. L. Ander, J. W. Michael, C. Benson, G. Jellita, K. o. Dalitso, J. S. Alexander, J. F.-T. Susan, S. G. Rosalind, A. K. Alexander and R. B. Martin (2013). "Soil-type influences human selenium status and underlies widespread selenium deficiency risks in Malawi." Scientific Reports.

Read, R., T. Bellew, J. G. Yang, K. E. Hill, I. S. Palmer and R. F. Burk (1990). "Selenium and amino acid composition of selenoprotein $\mathrm{P}$, the major selenoprotein in rat serum." The Journal of biological chemistry 265(29): 17899-17905. 
Romagné, F., D. Santesmasses, L. White, G. K. Sarangi, M. Mariotti, R. Hübler, A. Weihmann, G. Parra, V.

331 N. Gladyshev, R. Guigó and S. Castellano (2014). "SelenoDB 2.0: annotation of selenoprotein genes in 332 animals and their genetic diversity in humans." Nucleic acids research 42(Database issue): D437-443.

333 Saito, Y., N. Sato, M. Hirashima, G. Takebe, S. Nagasawa and K. Takahashi (2004). "Domain structure of

\section{bi-functional selenoprotein P." The Biochemical journal 381(Pt 3): 841-846.}

Santos, S., G. Ungureanu, R. Boaventura and C. Botelho (2015). "Selenium contaminated waters: An overview of analytical methods, treatment options and recent advances in sorption methods." Science of The Total Environment 521-522: 246-260.

Shetty, S. P., R. Shah and P. R. Copeland (2014). "Regulation of selenocysteine incorporation into the selenium transport protein, selenoprotein P." The Journal of biological chemistry 289(36): 25317-25326. Steinbrenner, H., E. Bilgic, L. Alili, H. Sies and P. Brenneisen (2006). "Selenoprotein P protects endothelial cells from oxidative damage by stimulation of glutathione peroxidase expression and activity." Free radical research 40(9): 936-943.

Sunde, R. A. and K. B. Hadley (2010). "Phospholipid hydroperoxide glutathione peroxidase (Gpx4) is highly regulated in male turkey poults and can be used to determine dietary selenium requirements." Experimental biology and medicine 235(1): 23-31.

Sunde, R. A., A. M. Raines, K. M. Barnes and J. K. Evenson (2009). "Selenium status highly regulates selenoprotein mRNA levels for only a subset of the selenoproteins in the selenoproteome "Bioscience Reports 29(5): 329-338.

Wedekind, K. J., S. Yu and G. F. Combs (2004). "The selenium requirement of the puppy." Journal of Animal Physiology and Animal Nutrition 88(9-10): 340-347.

Weiss Sachdev, S. and R. Sunde (2001). "Selenium regulation of transcript abundance and translational efficiency of glutathione peroxidase-1 and -4 in rat liver." The Biochemical journal 357(Pt 3): 851-858.

Weiss, S. L., J. K. Evenson, K. M. Thompson and R. A. Sunde (1996). "The selenium requirement for glutathione peroxidase mRNA level is half of the selenium requirement for glutathione peroxidase activity in female rats." Journal of Nutrition 126(9): 2260-2267.

Weiss, S. L., J. K. Evenson, K. M. Thompson and R. A. Sunde (1997). "Dietary selenium regulation of glutathione peroxidase mRNA and other selenium-dependent parameters in male rats." Journal of Nutritional Biochemistry 8(2): 85-91. 
Figure 1. The receptor binding sites and selenocysteine (Sec) residues of vertebrate selenoprotein P (SEPP1). From the N-terminal side, SEPP1 is comprised of a conserved Nterminal domain Sec residue, followed by several proposed heparin binding sites which include a histidine rich region. Following this, there is the shorter Sec residue rich $\mathrm{C}$-terminal domain which contains an APOER2 binding site. The C-terminal domain can be further divided into two subdomains. The first subdomain exists on the N-terminal side of the APOER2 binding site and contains a region with low conservation of Sec residues among vertebrates (mainly due to Sec to cysteine (Cys) conversions (Lobanov et al., 2008)). The second subdomain is located downstream of the APOER2 binding site and contains five Sec residues that are conserved across vertebrate species. Several species of amphibians also have an additional Sec residue in the Cterminal end of this region (Lobanov et al., 2008). The proposed heparin binding sites/histidine rich regions are based on rat SEPP1 found by Hondal et al. (2001). Similar histidine rich regions are found in the SEPP1s of other species (selenodb.org). Cys residues outside the C-terminal domain are not shown. Red lines = conserved Sec residues; Black lines = Cys or Sec residues; Green lines $=\mathrm{Cys} / \mathrm{Sec}$ residues within the APOER2 binding site; Green box grids $=$ proposed heparin binding sites.

Figure 2. The relationship between the selenocysteine content of selenoprotein $P$ and selenium requirements in vertebrates. The solid line with the solid circles $(\bullet)$ is the best fit model for the SEPP1 Sec content versus Se requirements (mg Se/kg dry matter (DM)) from 14 species with representatives from the mammalian, bird and bony fish classes where the genome sequences were available (Second order polynomial, $\mathrm{R}^{2}=0.78, \mathrm{y}=3.3+93 \mathrm{x}-175 \mathrm{x}^{2}$ ). The broken line represents the same data modelled with an additional five bony fish species with known Se requirement levels (O), but unannotated genomes. SEPP1 Sec content in these fish were assumed to be within the likely range of 15-17 Sec residues found for fish in general (5PL Asymmetric sigmoidal, $\left.\mathrm{R}^{2}=0.86, \mathrm{y}=-9.98+\left(26.9 /((1+10((-2.23397-\mathrm{X}) \times 4.661)))^{1.9^{\wedge} 10}\right)\right)$. Shaded boxes group animals within classes. The $\mathrm{X}$ axis is log transformed. 
Table $\mathbf{1}_{\text {(on next page) }}$

The Se requirements (mg Se/kg DM) predicted by the model (Figure 2, solid line) with changes in the selenocysteine (Sec) content of selenoprotein P (SEPP1).

${ }^{1} \mathrm{mg} \mathrm{Se} / \mathrm{kg}$ feed DM, mean $( \pm 95 \% \text { confidence interval, when shown })^{2}$ There are currently no known species with full length SEPP1 containing 6 Sec residues. 
1 Table 1. The Se requirements ( $\mathrm{mg} \mathrm{Se} / \mathrm{kg} \mathrm{DM}$ ) predicted by the model (Figure 2, solid line) with

2 changes in the selenocysteine (Sec) content of selenoprotein P (SEPP1).

\section{Class Sec no. $\quad$ Predicted Se}

\section{requirement $^{1}$}

\begin{tabular}{ccc}
\hline$?^{2}$ & 6 & $0.03 \pm 0.03$ \\
& 7 & $0.04 \pm 0.03$ \\
& 8 & $0.06 \pm 0.02$ \\
& 9 & $0.07 \pm 0.02$ \\
$\frac{0}{\pi}$ & 10 & $0.09 \pm 0.01$ \\
& 11 & $0.10 \pm 0.02$ \\
& 12 & $0.12 \pm 0.03$ \\
& 13 & $0.14 \pm 0.04$ \\
& 14 & $0.17 \pm 0.05$ \\
& 15 & $0.20 \pm 0.04$ \\
Bony & $16+$ & $>0.20$ \\
fish & & \\
\hline
\end{tabular}

$31 \mathrm{mg} \mathrm{Se} / \mathrm{kg}$ feed DM, mean ( \pm 95\% confidence interval, when shown)

$4 \quad{ }^{2}$ There are currently no known species with full length SEPP1 containing 6 Sec residues. 
The receptor binding sites and selenocysteine (Sec) residues of vertebrate selenoprotein P (SEPP1)

From the N-terminal side, SEPP1 is comprised of a conserved N-terminal domain Sec residue, followed by several proposed heparin binding sites which include a histidine rich region. Following this, there is the shorter Sec residue rich C-terminal domain which contains an APOER2 binding site. The C-terminal domain can be further divided into two subdomains. The first subdomain exists on the N-terminal side of the APOER2 binding site and contains a region with a low conservation of Sec residues among vertebrates (mainly due to Sec to cysteine (Cys) conversions ( Lobanov et al., 2008 )). The second subdomain is located downstream of the APOER2 binding site and contains five Sec residues that are conserved across vertebrate species. Several species of amphibians also have an additional Sec residue in the C-terminal end of this region ( Lobanov et al., 2008 ). The proposed heparin binding sites/histidine rich regions are based on rat SEPP1 found by Hondal et al. ( 2001 ). Similar histidine rich regions are found in the SEPP1's of other species (selenodb.org). Cys residues outside the C-terminal domain are not shown. Red lines = conserved Sec residues; Black lines $=$ Cys or Sec residues; Green lines $=$ Cys/Sec residues within the APOER2 binding site; Green box grids $=$ proposed heparin binding sites . 


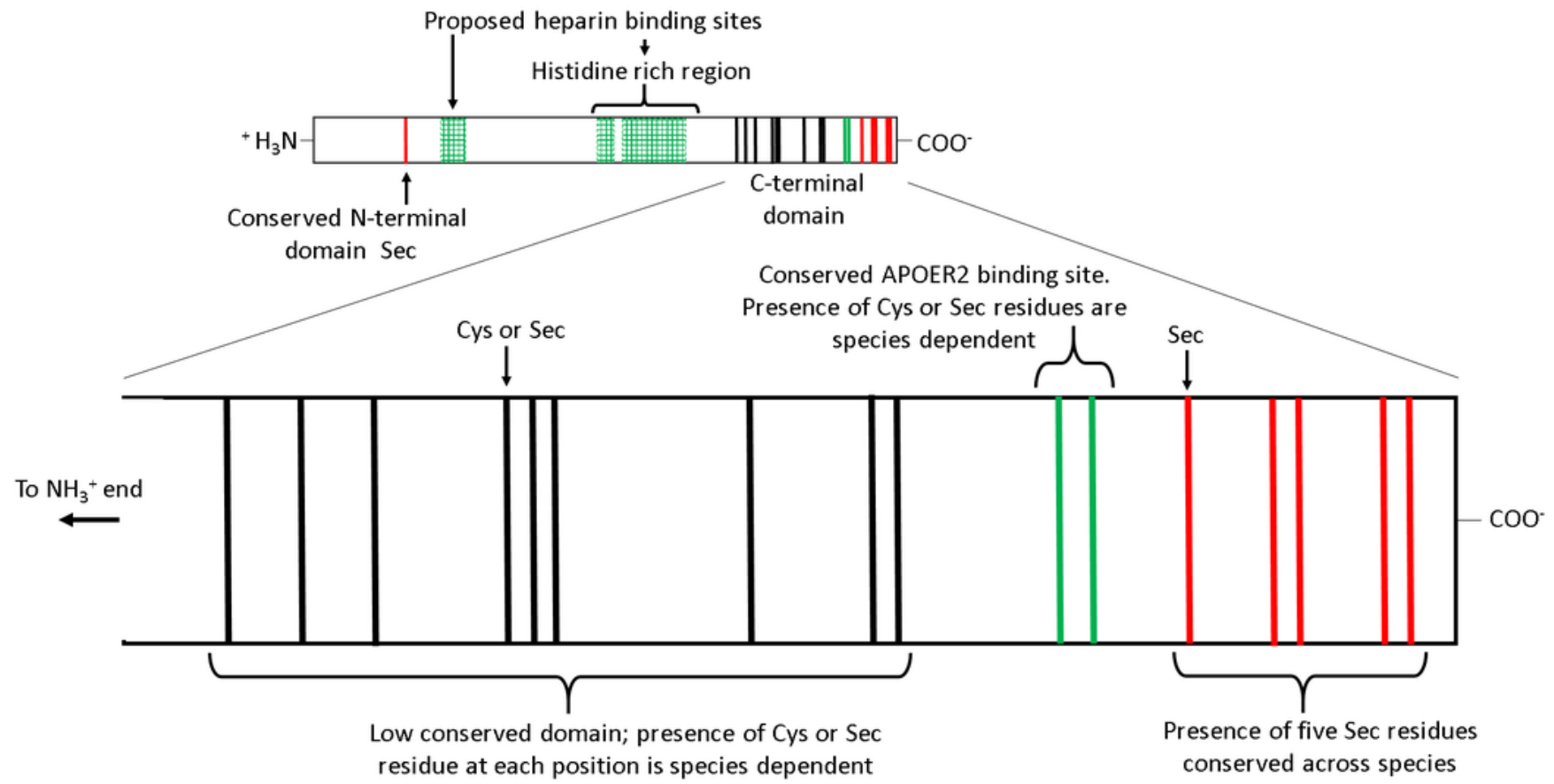




\section{Figure 2 (on next page)}

The relationship between the selenocysteine content of selenoprotein $\mathrm{P}$ and selenium requirements in vertebrates.

The solid line with the solid circles $(\bullet)$ is the best fit model for the SEPP1 Sec content versus Se requirements (mg Se/kg dry matter (DM)) from 11 species with representatives from the mammalian and bony fish classes where the genome sequences were available (Second order polynomial, $\left.R^{2}=0.88, y=4.3+78 x-122 x^{2}\right)$. The broken line represents the same data modelled with an additional five bony fish species with known Se requirement levels (O), but unannotated genomes. SEPP1 Sec content in these fish were assumed to be within the likely range of 15-17 Sec residues found for fish in general (5PL Asymmetric sigmoidal, $\mathrm{R}^{2}$ $\left.=0.92, y=5.13+\left(11.9 /((1+10(-1.6410-x) \times 6.391))^{9.611^{110}}\right)\right)$. Shaded boxes group animals within classes. The $X$ axis is log transformed. 
SEPP1

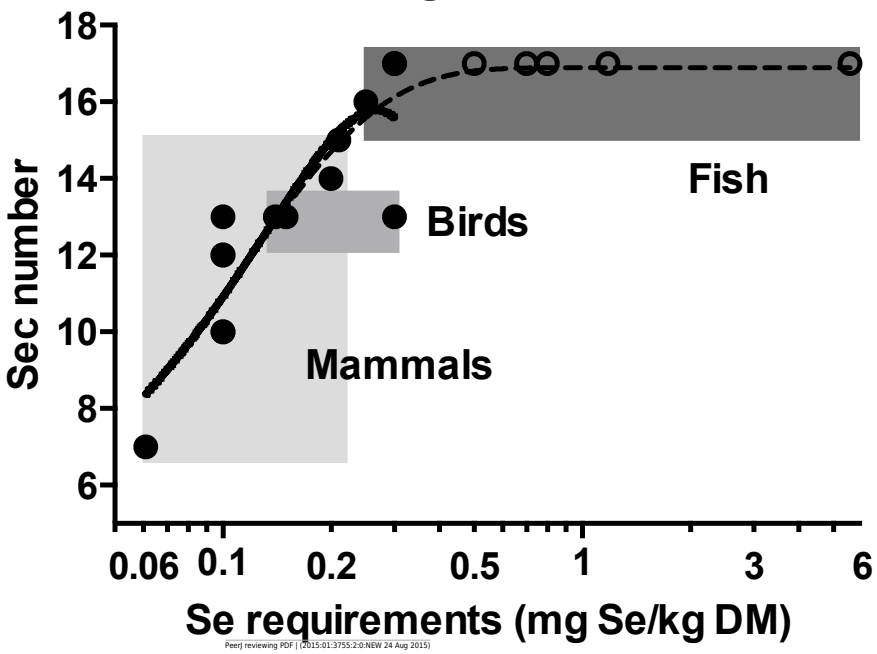

